bined with colloidal ion exchange resin can also be used for the destructive agent in stereotaxic brain surgery.

Method of preparing colloidal isotopes:

Adding water, anion exchange resin AMBERLITE IRA-400 $\left(\mathrm{R} \equiv \mathrm{Na}^{+} \mathrm{Cl}^{-}\right)$is ground in Ball-Mill and the colloidal fluid is obtained. The ion exchange between resin and isotope can be formulated as follows:

$$
\begin{aligned}
& \mathrm{R} \equiv \mathrm{Na}^{+} \mathrm{Cl}^{-}+\mathrm{H}_{3}{ }^{+} \mathrm{PO}_{4}{ }^{---} \underset{\leftarrow}{\longrightarrow} \equiv \mathrm{R}^{+} \mathrm{H}_{2}{ }^{32} \mathrm{PO}_{4}{ }^{-}+\mathrm{H}^{+} \mathrm{Cl}^{-} \\
& \mathrm{R} \equiv \mathrm{Na}^{+} \mathrm{Cl}^{-}+\mathrm{Na}^{+131} \mathrm{I}^{-} \rightleftarrows \mathrm{R} \equiv \mathrm{N}^{+131} \mathrm{I}^{-}+\mathrm{Na}^{+} \mathrm{Cl}^{-}
\end{aligned}
$$

$2.5 \mathrm{mC}$ of each ${ }^{32} \mathrm{P}$ or ${ }^{131} \mathrm{I}$ are combined with $100 \mathrm{mg}$ of Amberlite IRA-400 almost completely.

Experimental Method and Results:

The colloidal resin, $100 \mathrm{mg}$ in weight and $3 \sim 1,000 \mu \mathrm{C}$ in radioactivity, was injected into the globus pallidus of cats, with the aid of stereotaxic apparatus of Hess type. Following the injection general condition of the animal and the decrement of radio-activity in the brain were investigated. Histological examination of the brain at the various period after the injection was also performed.

The local radio-activity reduced to half in 24 hours in cases of ${ }^{131} I$ and in 3 days in cases of ${ }^{32} \mathrm{P}$, the reduction thereafter being in coincidence with natural decrement rate.

Animals never fell into coma. Sometimes motor paresis and disturbances in gait were seen, but recovered in several days.

Slight increase in body temperature was usually see for about ten days after the injection.

The leucocyte count, after showing slight increase for few days, dropped to about $50 \%$, and one month was necessary to regain the normal value. higher.

If the radio-activity exceeded $1 \mathrm{mC}$, the mortality became considerably

Macroscopic and histologic investigation revealed that the resin was injected in a mass into the target. Surrounding the injected resin, accummulation of compound molecular corpuscles and the narrow zone of disintegration of nervous tissue was observed.

From results obtained until now, it is our impression that the colloidal ion exchange resin combined with isotopes can be used as the destructive agent in stereotaxic brain surgery.

More detailed research must be continued in the future.

\title{
B7. Involuntary Disorders from an Aspect of Control System.
}

\section{SOBUE}

The 1st Dept. of Int. Med. Nagoya Univ. School of Med.

Servomechanism of movement is considered to have spacio-temporary factors. 
To study on the servomechanism of movement in various involuntary diseases compared with a normal state is a significant point in order to analyse the mechanism of the involuntary movement.

Fluctuation in amplitude of $\mathrm{H}$ wave examined by evoked EMG is more marked in involuntary diseases than normal subjects and is also apt to be influenced by various exteroceptive stimulations such as sensory or emotional. These similar results have also been shown in diencephalic diseases and temporal lesions. From these observations, it may be suggested that a large number of regions in central nervous system are related to the servomechanism of movement.

Muscular servomechanism on cases of Parkinson subjects was examined by a method of frequency response. Parkinson subjects showed lower gain curves in various frequency than normal subjects. On the contrary, curves produced from phase differences in each cycle are fairy similar to normal cases. Nyquist diagram obtained by the gain and phase differences showed rather stable pattern in Parkinson subjects than normal ones.

Dynamic aspects upon the mechanism of movement should be introduced in clinical treatments of involuntary disorders.

\title{
B8. About Experiments using Stereotaxic Instrument in Animals.
}

\author{
K. TAKAGI \\ Dept. of Physiol. Nagoya Univ. School of Med.
}

When pressure stimuli were applied to the skin of the animal, it fell into the state of 'hypnosis' and electroencephalographically showed patterns of low activity state of the brain.

Using rabbits with chronically implanted surface and deep electrodes, we observed changes of EEG patterns and skin pressure effects upon the brain activity during restrained conditions with stereotaxic fixing instrument.

Before beginning fixation, seven hinged paper holders were applied in the unrestrained condition and it was ascertained that EEG patterns turned from arousal to drowsy ones by clipping. From just after fixation of head and extremities by Noken type stereotaxic instrument, there occured the same changes in EEG patterns as induced by clipping before fixation. About $10 \mathrm{~min}$. after fixation when simultaneously recorded rectal temperature began to decline, there mingled arousal patterns intermittently. About 2 hours after fixation when rectal temperature reached about $3{ }^{\circ} \mathrm{C}$ lower than before fixation and kept constant value, arousal patterns continued as long as before fixation. Here seven hinged paper holders were applied again, but no effect was observed. About $30 \mathrm{~min}$. after fixation was released, the effect to the skin pressure stimuli began to appeare again, and rectal temperature reached that of before fixation, clipping effect also 